\title{
Antiproton Accumulator in the Main Injector Era
}

\author{
Vladimir Visnjic \\ Fermi National Accelerator Laboratory \\ P.O. Box 500, Batavia, Illinois 60510
}

September 1992 


\section{Disclaimer}

This report was prepared as an account of work sponsored by an agency of the United States Government. Neither the United States Government nor any agency thereof, nor any of their employees, makes any warranty, express or implied, or assumes any legal liability or responsibility for the accuracy, completeness, or usefulness of any information, apparatus, product, or process disclosed, or represents that its use would not infringe privately owned rights. Reference herein to any specific commercial product, process, or service by trade name, trademark, manufacturer, or otherwise, does not necessarily constitute or imply its endorsement, recommendation, or favoring by the United States Government or any agency thereof. The views and opinions of authors expressed herein do not necessarily state or reflect those of the United States Government or any agency thereof. 
TM-1797

September, 1992

\title{
Antiproton Accumulator in the Main Injector Era
}

\author{
Vladimir Visnjic \\ Fermi National Accelerator Laboratory \\ Batavia, IL 60510
}

\begin{abstract}
I propose a new lattice for Fermilab Antiproton Accumulator. The lattice is designed with the aim of implementing higher bandwidth stochastic cooling systems, thus enabling the Accumulator to handle antiproton beam intensities expected in the Main Injector era. The design proposed here satisfies very well all the requirements for the new Accumulator concerning the lattice functions, stochastic cooling parameters, and the tunability of the machine.
\end{abstract}




\section{INTRODUCTION}

With the advent of Main Injector ${ }^{1}$ the number of antiprotons injected into Fermilab Antiproton Accumulator is expected to increase about three times, while at the same time the period between injections should decrease from 2.4 to $1.5 \mathrm{sec}$. Since the cooling rate is fundamentally proportional to the bandwidth of the system divided by the number of particles in the beam, this will inevitably lead to slowing down the rate at which the antiproton beam is cooled. Qualitatively speaking, both the stack tail and the core will become denser and it will take longer to push particles into the core and to cool the core down to useful emittances.

The solution is in the bandwidth increase of the stochastic cooling electronics. Presently, the stack tail systems use the 1-2 $\mathrm{GHz}$ band, while the core whose smaller frequency spread permits the use of higher bandwidth uses $2-4 \mathrm{GHz}$. There is also a $4-8 \mathrm{GHz}$ core momentum cooling system. An upgrade to twice these values is called for. This, however, requires changing the lattice of the machine which can be seen as follows. The width of Schottky bands is proportional to their frequency, thus increasing the bandwidth by a factor $n$ will result in $n$ times wider Schottky bands. Since the bands are not allowed to overlap inside the amplifier bandwidth, the frequency spread $\Delta f / f$ of the beam must be decreased in the same proportion. On the other hand, the frequency spread of the beam is related with the momentum spread as

$$
\frac{\Delta f}{f}=\eta \frac{\Delta p}{p} .
$$

For a given beam energy, $\eta$ depends on $\gamma_{T}$ of the lattice,

$$
\eta=\frac{1}{\gamma_{T}^{2}}-\frac{1}{\gamma^{2}},
$$

and therefore the lattice must be changed if one changes the bandwidth.

Inverting formula (1) gives $\gamma_{T}=\left(1 / \gamma^{2}+\eta\right)^{-1 / 2}$, shown in Fig.1 for the Accumulator energy $\left(1 / \gamma^{2}=0.011\right)$. Presently, $\eta=0.023$ and $\gamma_{T}=5.43$. Note that only the absolute value of $\eta$ is important, thus for each desired numerical value of $\eta$ we can entertain two possibilities. For the present value $|\eta|=0.023$, the positive value leads to the real $\gamma_{T}=5.43$, while $\eta=$ -0.023 requires an imaginary $\gamma_{T}$ lattice with $\gamma_{T}=i 9.13$. The latter has not been considered, but it might have been an interesting possibility since the transition crossing in the charmonium experiment E760 would have been avoided. Doubling the bandwidth requires $|\eta|$ of 0.011 . The solutions are $\gamma_{T}=6.74$ for positive $\eta$ and $\gamma_{T}=\infty$ for negative one. The latter value would almost certainly lead to too low dispersion for the purpose of stochastic cooling and will not be considered further. 


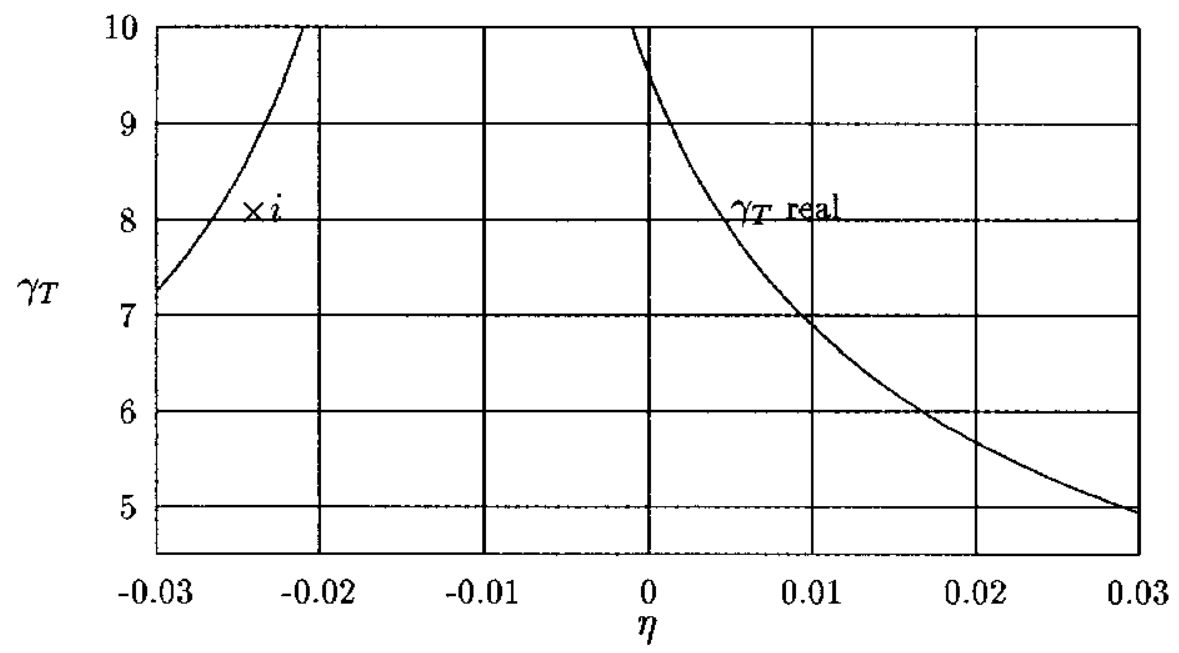

Fig. 1 Transition $\gamma$ as function of $\eta$ in the Accumulator $\left(E_{k}=7.9 \mathrm{GeV}\right)$.

\section{NEW ACCUMULATOR LATTICE}

One can show that

$$
\frac{1}{\gamma_{T}^{2}}=\frac{1}{C} \oint_{C} \frac{\eta_{x}(s)}{\rho(s)} d s,
$$

where $\eta_{x}, \rho$, and $C$ are the dispersion, the bending radius and the circumference of the machine, respectively. Since the integrand is non-zero only in the dipoles, one concludes that $\gamma_{T}$ is determined by the values of the dispersion function in the dipoles. In fact, $\gamma_{T}$ can be approximately evaluated by means of the expression

$$
\frac{1}{\gamma_{T}^{2}} \approx \frac{1}{C} \sum_{\text {dipoles }}<\eta_{x}>_{i} \theta_{i}
$$

where $\left\langle\eta_{x}\right\rangle_{i}$ is the average dispersion in a given dipole and $\theta_{i}$ its bend angle. From these expressions it follows that in order to increase $\gamma_{T}$ we have to decrease values of dispersion in the dipoles. At the same time, dispersion in the straight sections where the pickups and the kickers are located must not change significantly. The lattice functions of one superperiod of the present Accumulator are shown in Fig. 2. On top of the picture is a schematic representation of the lattice, with the height of the boxes representing the field gradient.

The lattice presented here has the attractive feature of being based on the same geometry as the present Accumulator. It has been obtained by varying the fields in the quadrupoles in such a way as to diminish the dispersion in the region of large dipoles, subject to the constraints of (1) maintaining its value in the high- and low dispersion sections, (2) maintaining the values of 


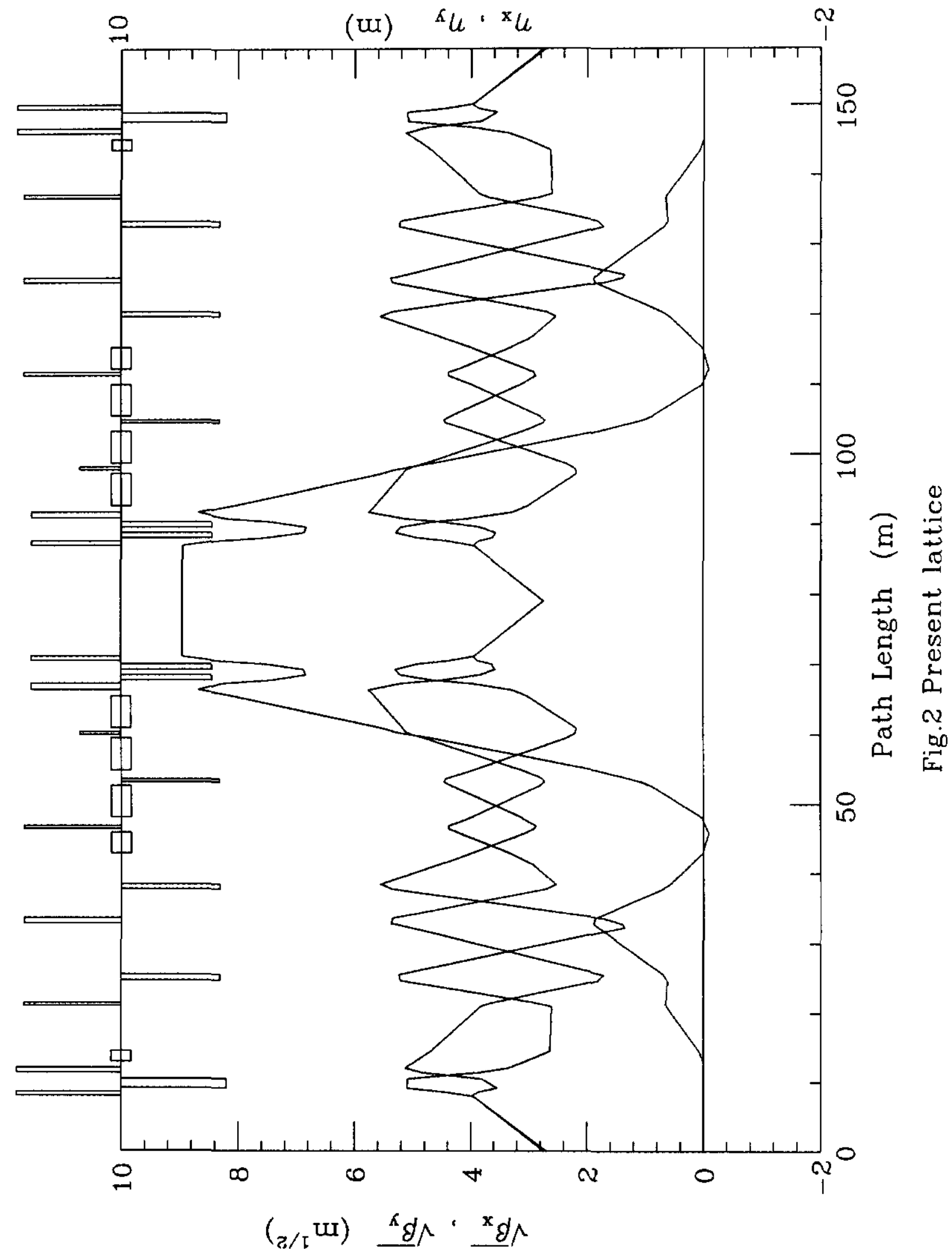


beta functions within reasonable limits, and (3) keeping the beta functions in low-beta regions as small as possible. In addition, there is an important constraint imposed by betatron stochastic cooling, both stack tail and core, namely that the phase advance between the pickups and the kickers must be close to an odd multiple of $\pi / 2$. The lattice obtained in this way is shown in Fig. 3. A similar investigation was done by G. Dugan ${ }^{2}$ who arrived at the solution shown in Fig. 4. These two solutions are quite different and are summarized (together with the present lattice) in the following table:

\begin{tabular}{|c|c|c|c|}
\hline PARAMETER & NOMINAL LATTICE & LATTICE FROM Ref.2 & NEW LATTICE \\
\hline $\mathrm{Q} 1(\mathrm{~T} / \mathrm{m})$ & 10.38 & 8.12 & 13.892 \\
$\mathrm{Q} 2(\mathrm{~T} / \mathrm{m})$ & -10.38 & -10.06 & -10.536 \\
$\mathrm{Q} 3(\mathrm{~T} / \mathrm{m})$ & 10.38 & 11.41 & 8.616 \\
$\mathrm{Q} 4(\mathrm{~T} / \mathrm{m})$ & 9.66 & 5.35 & 10.483 \\
$\mathrm{Q} 5(\mathrm{~T} / \mathrm{m})$ & -9.74 & -9.07 & -8.792 \\
$\mathrm{Q} 6(\mathrm{~T} / \mathrm{m})$ & 9.66 & 10.44 & 10.116 \\
$\mathrm{Q} 7(\mathrm{~T} / \mathrm{m})$ & -9.74 & -9.54 & -9.404 \\
$\mathrm{Q} 8(\mathrm{~T} / \mathrm{m})$ & 9.66 & 11.03 & 9.310 \\
$\mathrm{Q} 9(\mathrm{~T} / \mathrm{m})$ & -9.74 & -6.91 & -9.531 \\
$\mathrm{Q} 10(\mathrm{~T} / \mathrm{m})$ & 4.087 & 6.34 & 3.045 \\
$\mathrm{Q} 11(\mathrm{~T} / \mathrm{m})$ & 8.94 & 3.91 & 9.043 \\
$\mathrm{Q} 12(\mathrm{~T} / \mathrm{m})$ & -8.94 & -6.83 & -10.128 \\
Q13(T/m) & -8.94 & -6.83 & -8.487 \\
Q14(T/m) & 8.94 & 10.47 & 9.999 \\
$\gamma_{T}$ & 5.43 & 7.07 & 6.76 \\
$\eta$ & 0.023 & 0.009 & 0.011 \\
$\nu_{x}$ & 6.61 & 6.67 & 6.76 \\
$\nu_{y}$ & 8.61 & 7.51 & 7.967 \\
$\phi_{\mathrm{P}}-\mathrm{K}\left(\frac{\pi}{2}\right)$ & 8.82 & 8.88 & 9.0 \\
$\beta_{x}(\mathrm{~A} 10)(\mathrm{m})$ & 7.6 & 7.5 & 2.5 \\
$\beta_{y}(\mathrm{~A} 10)(\mathrm{m})$ & 7.3 & 7.2 & 4.8 \\
$\beta_{x}(\mathrm{~A} 20)(\mathrm{m})$ & 7.6 & 7.5 & 5.5 \\
$\beta_{y}(\mathrm{~A} 20)(\mathrm{m})$ & 7.5 & 7.6 & 8.2 \\
$\eta_{x}(\mathrm{~A} 10)(\mathrm{m})$ & 0.0 & 0.02 & -0.39 \\
$\eta_{x}(\mathrm{~A} 20)(\mathrm{m})$ & 8.9 & 10.9 & 9.1 \\
Max $\beta_{x}(\mathrm{~m})$ & 33.2 & 35.0 & 37.6 \\
Max $\beta_{y}(\mathrm{~m})$ & 30.9 & 33.8 & 38.2 \\
Max $\beta_{y}$ & & & 19.3 \\
in dipoles $(\mathrm{m})$ & 20.0 & 26.673 & \\
\hline
\end{tabular}

TABLE 1 Properties of the present Accumulator lattice, the one proposed in Ref. 2 and the new one. 


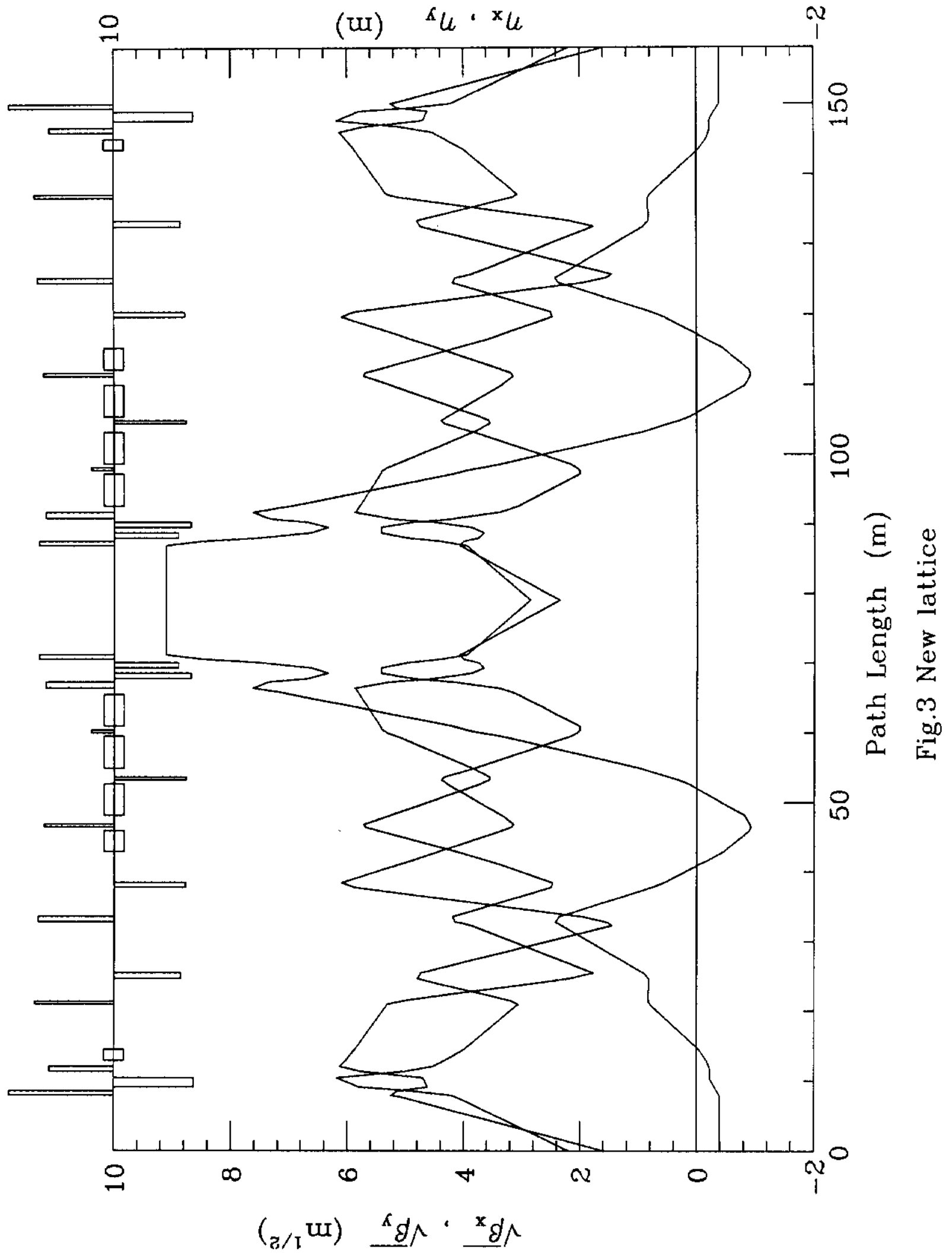




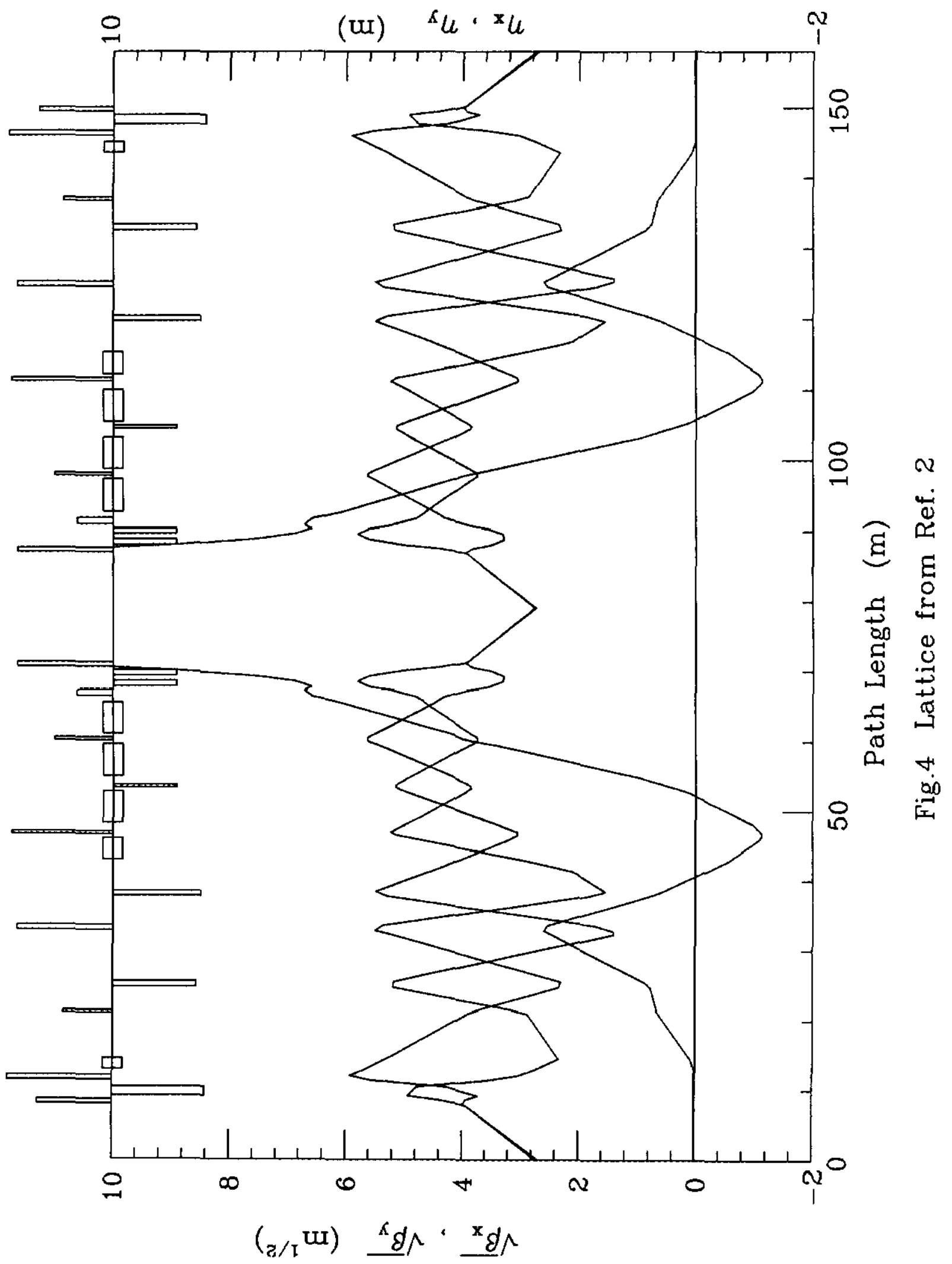


We can point out certain advantages of this design compared with the one proposed in Ref. 2. First and most important, the value of $\gamma_{T}$ is exactly as required, while in [2] it is higher than needed, leading to an unnecessarily small $\eta$ ( $\eta$ should be as large as possible without causing Schottky bands to overlap). Second, $\beta_{x}$ in the high dispersion straight sections is here about $30 \%$ smaller, which is a welcome feature for stochastic cooling. At the same time the values of $\beta_{\max }$ are only slightly increased. More important, the value of $\beta_{y \max }$ in the dipoles, which is of most concern, is actually smaller in the new lattice than in both the present one and the one from Ref. 2 . In the same way $\beta_{x}$ in the zero dispersion straight section is smaller by a factor of 3 . The small negative dispersion $(-39 \mathrm{~cm})$ in the low dispersion straight section is intentional and based on the experience with the present design where there is a small positive "residual" dispersion although the design value is zero (see Fig. 2). The beam size is a combined effect of these two:

$$
\sigma_{x}=\sqrt{\epsilon_{x} \beta_{x}+\left(\eta_{x} \frac{\Delta p}{p}\right)^{2}}
$$

Fig. 5 shows the beam size in the low dispersion straight section as a function of emittance for the three lattices. Here the momentum spread is $0.79 \%$ as required by stacking. Note that this assumes the design values for the lattices. In reality, the nominal lattice has a small positive dispersion and the lattice proposed here should have essentially no dispersion in the low-dispersion sections and the beam size should actually be smaller in the new lattice for all the values of the emittance.

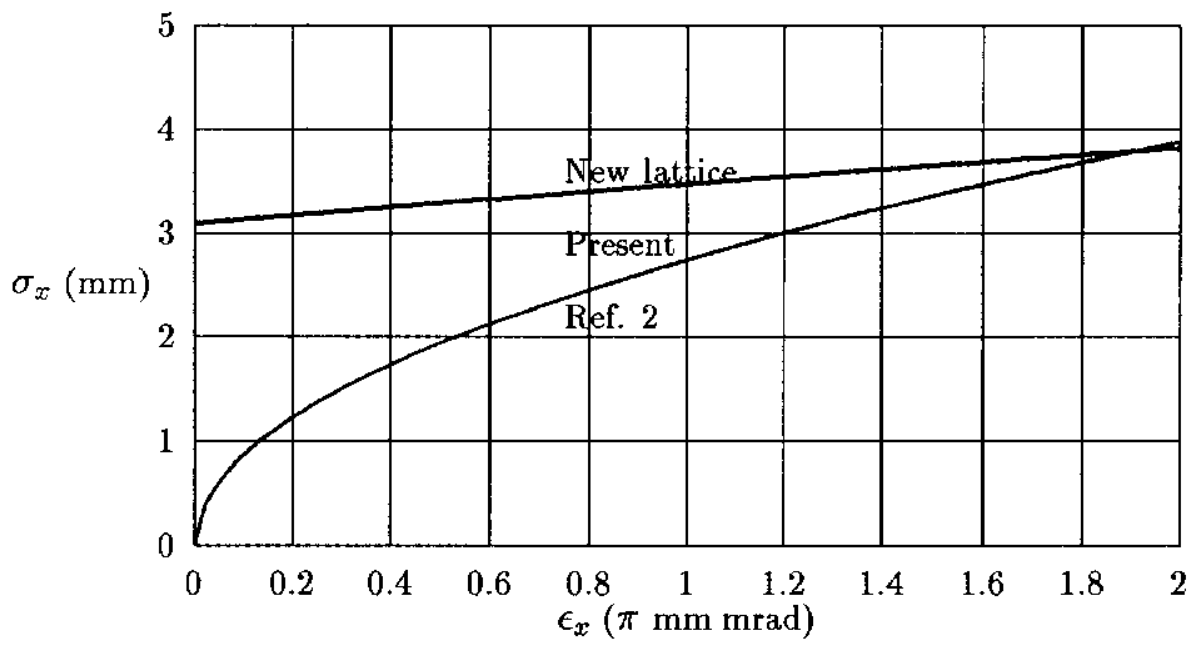

Fig. 5 Beam size in low dispersion straight section for the new lattice, the present one and the one from Ref. 2 . The curves for the latter two differ only slightly. $\frac{\Delta p}{p}=0.79 \%$, as required by stacking. 
The new lattice offers also a small improvement in the betatron cooling rate: the pickup-kicker phase advance for betatron cooling is right on the mark being $9.0 \times \pi / 2$. Since the betatron cooling rate is proportional to $\sin \left(\phi_{P U-K}\right)$, it appears that in the new lattice it is expected to be $4 \%$ higher than in the present one and $2 \%$ higher than in the one from Ref. 2. Last but not least, the new lattice requires somewhat smaller increases in the field gradients than the one of Ref. 2. The largest increase in the latter is for the quadrupole Q10 which is required to run at $55 \%$ higher current than presently. In the new lattice, the highest increase is about $34 \%$ (quadrupole $\mathrm{Q}(1)$ ). The number of magnets whose currents are to be decreased or not increased by more than $5 \%$ is 10 for the new lattice, $v s$. 9 for the one of Ref. 2. This may be important for the field quality.

\section{TUNING}

The new lattice is tunable over a large range using the four existing quadrupole buses. An example of tuning is shown in Fig. 6. In this example, a $2 \%$ decrease in the QT bus (quadrupoles Q1, Q2 and Q3) results in the tunes $\nu_{x}=6.69$ and $\nu_{y}=7.89$ without noticeably changing any other property of the lattice. Finding a good working point for the new Accumulator will be the subject of a separate study.

\section{SUMMARY AND OUTLINE OF FUTURE STUDY}

The lattice proposed here satisfies all the basic requirements for the new Accumulator. The list of things that remain to be done includes:

1. Feasibility study of the present proposal concerning various aspects of running the quadrupoles at the proposed set of currents, like saturation, magnet cooling etc. It may turn out that some of the magnets will have to be replaced by new ones. Results of a preliminary feasibility study are reported in Ref. 3.

2. Finding the working point of the machine and doing tracking study. Study of resonances, aperture limits, placement of sextupoles etc.

3. Designing the changes that need to be done on the beam lines injecting beam into and extracting it from the Accumulator, such that the new Accumulator fits smoothly into the existing system.

4. As pointed out by Dugan, ${ }^{2}$ the implications of the change in $\eta$ on the RF system should be studied.

5. Study of stochastic cooling in the new lattice. Some of the lattice functions (for example, dispersion in low-dispersion straight section) may have to be changed somewhat from the values given here. 
I would like to thank C. Ankenbrandt, M. Church and J. Marriner for comments.

\section{REFERENCES}

${ }^{1}$ Fermilab Main Injector, Conceptual Design Report, 1992.

${ }^{2}$ G. Dugan, PBAR Note \# 484, 1989.

${ }^{3}$ V. Visnjic, PBAR Note \# 530, 1992. 


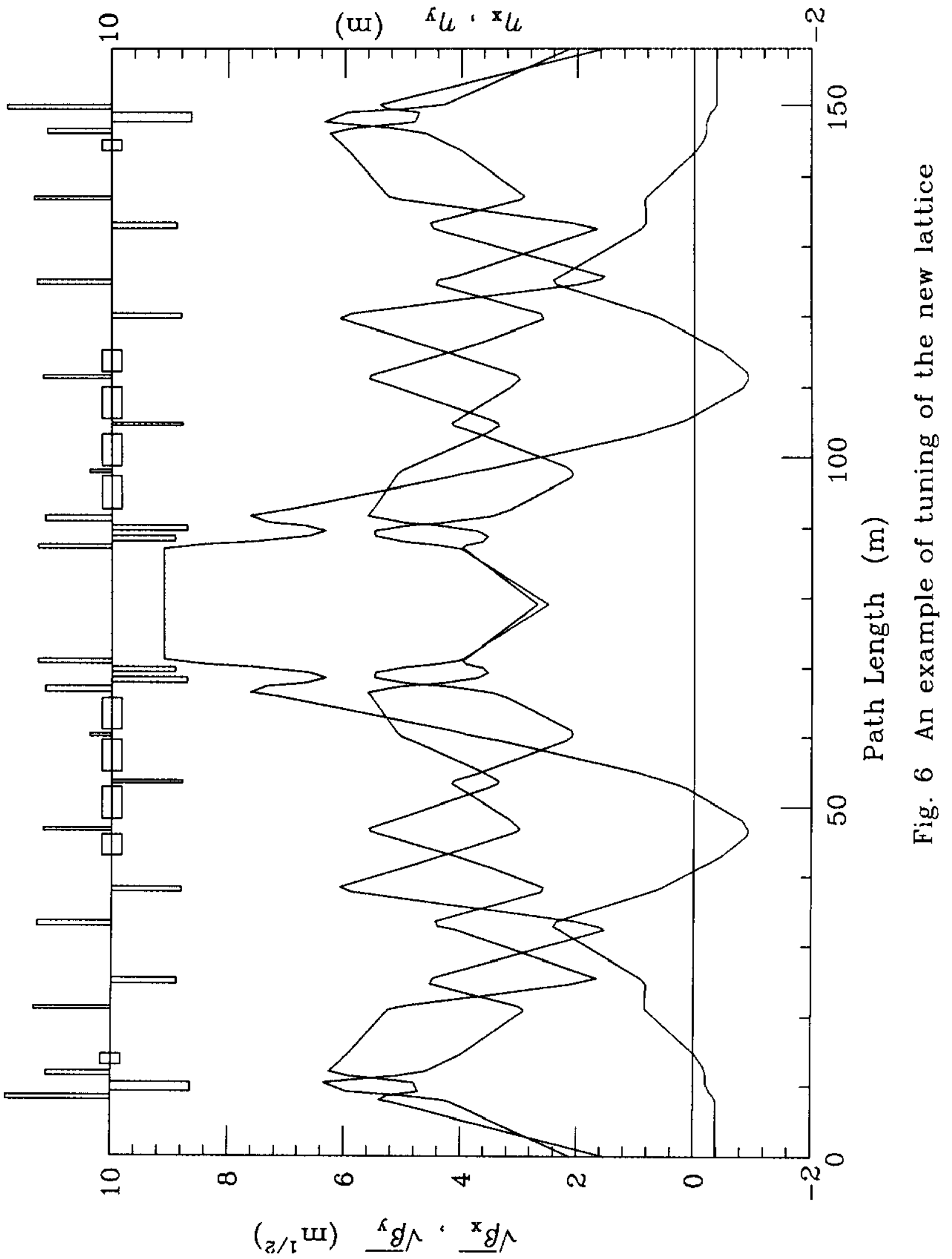

